\title{
Ultrafast Point Projection Electron Microscopy
}

\author{
Pratistha Shakya and Brett Barwick
}

Department of Physics, Trinity College, Hartford, Connecticut, USA

By combining the techniques of point projection electron microscopy with an ultrafast field emission tip source a simple compact ultrafast electron microscope can be constructed.

The microscope uses a tungsten field emission tip as an electron source and by focusing a femtosecond laser pulse onto the tip ${ }^{1,2}$ electron pulses with 100 fs are created. A schematic of the microscope setup is shown in Fig. 1. The microscopes operation can be switched to a standard CW mode by increasing the DC field applied to the tip. The magnification, $\mathrm{M}$, of the microscope ${ }^{3}$ is directly related to the ratio of the tip-to-specimen distance $\mathrm{d}$ and the distance from the tip to the detector D by,

$$
\mathrm{M}=\frac{D}{d}
$$

In our apparatus the distance $\mathrm{D}$ is fixed at $0.1 \mathrm{~m}$, however, $\mathrm{d}$, can be varied from $10^{-2} \mathrm{~m}$ to $10^{-5} \mathrm{~m}$ giving magnifications of $10 \times$ to $10,000 \times$.

To test the capabilities of the microscope, a Holey Carbon Quantifoil TEM grid was imaged. It has holes of different shapes and sizes with the smallest having a diameter of 1 micron. The images seen on the electron detector screen are shown in Fig. 2. For Fig. 2a, the tip voltage is -84 $\mathrm{V}$, and there is a large signal entirely due to $\mathrm{CW}$ electron emission. When the tip voltage is reduced to $-63 \mathrm{~V}$, there is almost zero $\mathrm{CW}$ emission and then by irradiating the tip with femtosecond laser pulses an image can be made using femtosecond electron pulses which is shown in Fig. 2b. We investigated the temporal duration of the electron emission from the tip using an autocorrelation experiment ${ }^{2}$ and found that the electron pulses are $\sim 100$ fs in duration. We also show that at low tip voltages the relatively low kinetic energy of the electrons results in electrostatic lensing near the specimen, which can cause the electrons to be deflected, as shown in Fig. 2d. While the deflections in Fig. 2d are due to the charging of the TEM grid, the images prove the sensitivity of the microscope to weak electric fields. While normally electrostatic distortions are considered detrimental to image formation in a conventional high energy TEM, we plan to use this sensitivity to follow the dynamics of ultrafast laser induced fields.

Combining the techniques of a point projection microscope with an ultrafast field emission source gives the microscope some advantages over traditional electron microscopes. Because of the very small distances between the electron source and sample the electron pulse has no time to broaden or disperse. This may allow temporal resolutions on the 10 femtoseconds to be attained, while also having spatial resolution better than $\sim 10 \mathrm{~nm}$ at low tip voltages ${ }^{3}$. This high temporal and spatial resolution will allow us to follow dynamics in a number of nanoscale systems. 
References:

[1] B. Barwick, J. Handali, and E. Quinonez, "Femtosecond photoelectron point projection microscope”, Review of Scientific Instruments 84, 103710 (2013).

[2] B. Barwick, C. Corder, J. Strohaber, N. Chandler-Smith, C. Uiterwaal, and H. Batelaan, "Laser-induced ultrafast electron emission from a field emission tip", New Journal of Physics 9, 142 (2007).

[3] V. T. Binh, V. Semet, and N. Garcia, "Low-energy-electron diffraction by nano-objects in projection microscopy without magnetic shielding”, Applied Physics Letters 65(19), 24932495 (1994).

[4] The authors acknowledge funding from Connecticut Space Grant and Trinity College FRC Grant.

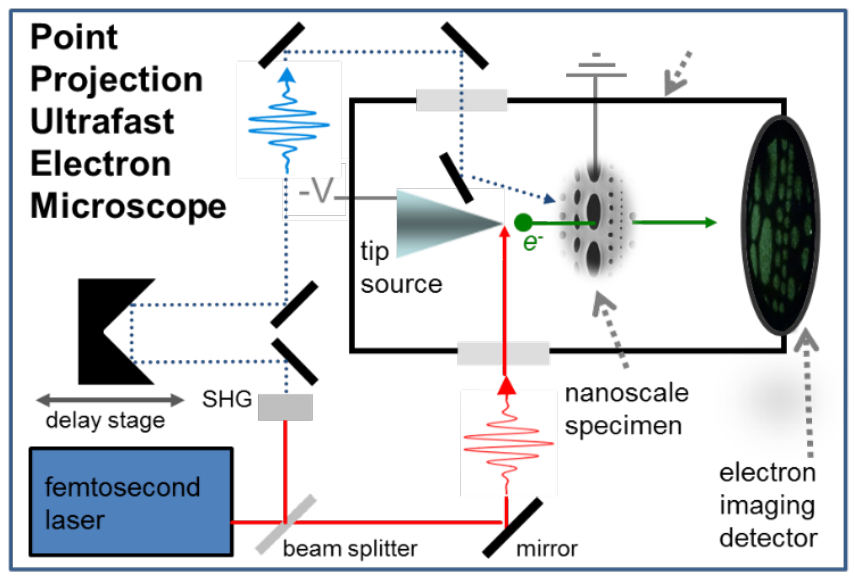

Fig. 1. Setup for the femtosecond point projection microscope. The probe excitation beam (red wave) is used to excite the field emission tip, producing pulsed electron packets. The pump beam (blue wave) is used to excite the sample. The pump and the probe laser beams enter the microscope from opposite sides. The pulsed electron packets are accelerated to the sample and the specimen image is then projected on the screen.

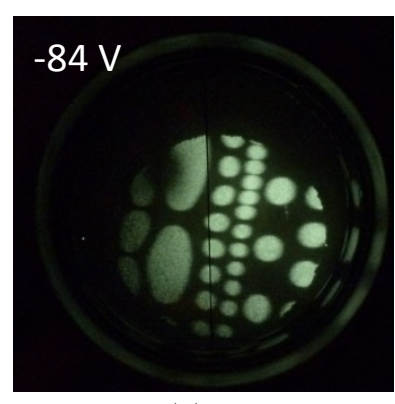

(a)

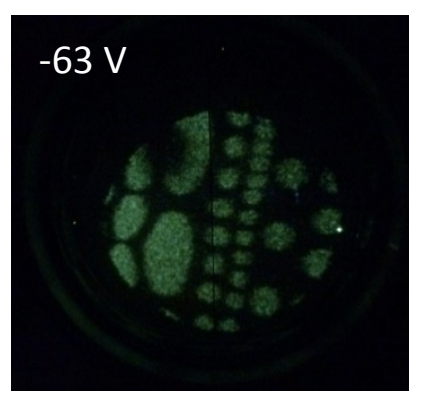

(b)

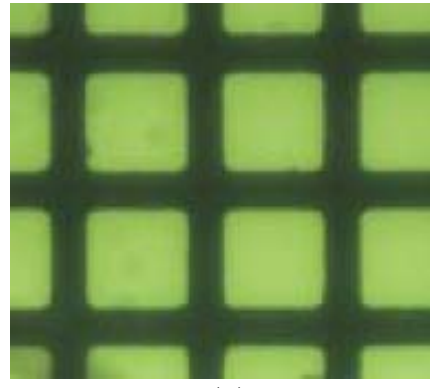

(c)

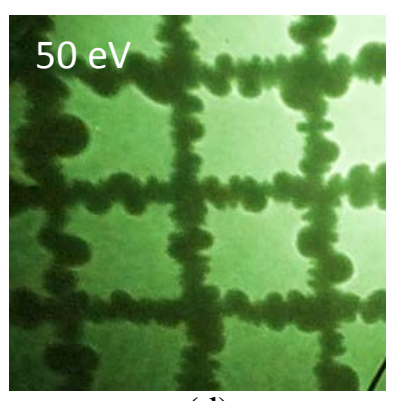

(d)

Fig. 2. CCD camera images of different TEM grids when operating the microscope with DC emission (a) and with femtosecond pulsed laser (b, d). Images (a) and (b) were taken in 2000× magnification. The CW mode required a tip voltage of $-84 \mathrm{~V}$ and the pulsed laser mode required $-63 \mathrm{~V}$ on the tips. Figure 2 (c) is an optical micrograph of a $120 \mu \mathrm{m} \times 120 \mu \mathrm{m}$ TEM grid at $63 \times$ magnification. Figure 2(d) was taken at the same magnification to show distortion on a TEM grid due to electrostatic lensing. This effect can be reduced by coating the specimen with materials of higher conductivity. 